Cinémas

Revue d'études cinématographiques

Journal of Film Studies

\title{
Narcissisme et corps spectatoriel
}

\section{Denis Bellemare}

Volume 7, numéro 1-2, automne 1996

La Représentation du corps au cinéma

URI : https://id.erudit.org/iderudit/1000931ar

DOI : https://doi.org/10.7202/1000931ar

Aller au sommaire du numéro

Éditeur(s)

Cinémas

ISSN

1181-6945 (imprimé)

1705-6500 (numérique)

Découvrir la revue

Citer cet article

Bellemare, D. (1996). Narcissisme et corps spectatoriel. Cinémas, 7(1-2), 37-54. https://doi.org/10.7202/1000931ar

\section{Résumé de l'article}

À partir de deux textes, "Aller au cinéma " de Metz et « En sortant du cinéma » de Barthes, l'auteur se pose la question du statut du corps spectatoriel. Le corps oublié du spectateur au profit du corps des personnages s'avère le lieu du travail du hors-champ et aussi de l'inconscient. En un tel lieu, toutes les identifications sont possibles et le spectateur n'en sort pas toujours gagnant, travaillé qu'il est par des forces antinomiques répressives, réversibles. 


\title{
Narcissisme et corps spectatoriel
}

\section{Denis Bellemare}

\begin{abstract}
RÉSUMÉ
A partir de deux textes, "Aller au cinéma" de Metz et "En sortant du cinéma" de Barthes, l'auteur se pose la question du statut du corps spectatoriel. Le corps oublié du spectateur au profit du corps des personnages s'avère le lieu du travail du hors-champ et aussi de l'inconscient. En un tel lieu, toutes les identifications sont possibles et le spectateur n'en sort pas toujours gagnant, travaillé qu'il est par des forces antinomiques répressives, réversibles.
\end{abstract}

\section{ABSTRACT}

Using two texts as a starting point, Metz's "Aller au cinéma" and Barthes' "En sortant du cinéma," the author poses the question of the status of the spectatorial body. The body of the spectator, overlooked in favour of the bodies on the screen, is revealed as the site of work outside the frame and also in the unconscious. In such a site, all identifications are possible, and the spectator doesn't always come out a winner, having been worked over by reversible antinomic forces.

C'est à partir de deux textes anciens que j'ai l'intention d'aborder les diverses articulations du corps spectatoriel: "Aller au cinéma" de Christian Metz, "En sortant du cinéma" de Roland Barthes. Le premier moule un creux réceptif où «la machine extérieure (le cinéma comme industrie) et la machine intérieure (la psychologie du spectateur)" (Metz, 1977, p. 14) nouent socialement avec les films de bonnes relations d'objet; ce texte de Metz instaure symboliquement l'Idéal du moi spectatoriel. Le second met en relief "le travail invisible des affects 
possibles" (Barthes, p. 105) d'une situation préhypnotique du cinéma. Il ne s'agit plus seulement d'un creux où se lover dans une bonne relation d'objet, mais encore d'un vide à remplir dans une sorte de capture de l'image; ce texte de Barthes fonde l'Imaginaire de la fameuse relation duelle du Moi idéal du spectateur de cinéma. Barthes en engage encore plus fondamentalement la dualité: "Comme si j'avais deux corps en même temps: un corps narcissique qui regarde, perdu dans le miroir proche, et un corps pervers, prêt à fétichiser, non l'image, mais précisément ce qui l'excède" (Barthes, p. 106). Le Moi spectatoriel n'est pas qu'un, il est peut-être double, probablement duel, certainement autre.

De l'entrée à la sortie de la salle de cinéma, que s'est-il réellement et imaginairement passé ? En aval et en amont du cinéma, de l'institution cinématographique au sujet spectateur, du champ des diverses conditions de représentation filmique au contrechamp de leurs modèles démultipliés, j'ai opté pour un corps sempiternellement présent et obligatoirement absent, à la fois voué et condamné au hors-champ du film, creux à habiter et vide à combler: le corps du spectateur. Ce corps de passage, traversé par la multiplicité et la clarté des discours, ne se donne à percevoir que dans le noir de la salle de cinéma, que dans l'opacité et l'intervalle des images, que dans la duplicité narcissique et perverse de ses positions.

\section{Corps}

Avant d'aborder le corps du sujet spectatoriel, ne vaut-il pas mieux en un premier temps décrire le corps propre du sujet (avant même qu'il ne soit spectateur), corps qui laisse déjà poindre de ses expériences du visible et du visuel de la perception un espace de figurabilité? Le corps propre s'avère ce lieu de perception, de fantasme et aussi de rêve où s'amortissent les dualités au profit de figures-synthèses, il sait unir dans sa représentation ce qui est séparé dans le processus. "Le corps est ce pouvoir originel de projection implicite dans la séparation du sujet et de l'objet et qu'il faut déduire à partir des traces laissées par l'écran du réel" (Sami-Ali, p. 84). Le corps comme schéma de représentation acquiert ainsi un statut métapsychologique rassemblant et 
médiatisant les diverses traces du processus de la perception et de la projection.

Ce concept de corps revient fréquemment dans de multiples écrits psychanalytiques dans l'enchaînement visuel et progressif d'éléments faisant rappel au dispositif cinématographique: de l'image à la projection sur une surface-écran. Il a l'attrait fondamental pour nous de concilier dans notre démarche interdisciplinaire le rôle ou plus précisément les fonctions diverses et stratifiées du visuel dans la représentation psychique par son passage de la psychanalyse à l'étude du cinéma. Les multiples articulations du réel et de l'imaginaire dans le schéma de représentation du corps propre dans une dynamique et une économique de forces opposées ne peuvent que nous rappeler certains processus du travail du film.

\section{- FORCES ANTINOMIQUES}

André Green, citant Le Moi et le Ça de Freud, place le Moi corporel au centre des distributions et des répartitions des représentations d'affects dans le système de perception visuelle: "Le Moi, rappelle Freud, est avant tout un Moi corporel, mais il ajoute: "Il n'est pas seulement un être de surface, il est luimême la projection d'une surface. "Cette précision nous aide à comprendre le rôle du regard et du miroir" (Green, p. 40). La contribution de Serge Leclaire dans Démasquer le réel complète le scénario psychique en relevant de ce lieu du corps de nombreux conflits: "Tel est le corps: un ensemble de lieux où l'ordre se démontre conflictuel. Le lieu s'y révèle pour ce qu'il est, la rencontre ou la coïncidence de forces antinomiques" (p. 62).

\section{— RÉVERSIBILITÉ}

Si les forces antinomiques viennent dire l'enjeu économique des conflits en présence dans la représentation du corps, le destin des pulsions viendra signifier dynamiquement la source et la direction de ses représentations d'affects. Le propre de cette dynamique n'engage pas un cheminement frontal, direct, univoque, au contraire. Cette activité poursuit un parcours plus complexe. Les pulsions destinées à la représentation du corps mettent en jeu l'identité narcissique et cette dernière peut s'opérer 
dans le renversement des pulsions en leur contraire et le retournement sur la personne proposée. Ce double retournement montre bien la réversibilité dynamique des représentations en cours, leur figurabilité sous une forme sans cesse retournée et renversée.

Il en est ainsi de la structure concentrique de la représentation du corps incluant un espace qui l'influe à son tour, et en cela, cette représentation présente une certaine réversibilité. L'image du corps comme unité de surface écranique d'inscription interpelle la complexité de fond de sa propre structure: le corps de l'image. Elle devient le support, comme on le dit du film, des mouvances psychiques de la représentation. Entre système totalisateur et instance, ce concept opératoire s'érige en instance constituée, constituante et constructrice et n'implique aucunement un rapport direct au monde. Et en ce sens, les modèles et les conditions de représentation des personnages au cinéma doivent non seulement être compris dans leur construction sociale et idéologique et leur construction discursive dans le récit, mais encore dans leur production cinématographique où, au contraire du récit du monde et du monde du récit, les positions psychiques s'avèrent labiles, antinomiques, réversibles.

Il en est ainsi du corps filmique, entre le film et le monde. Le film comme opération médiatrice certes, mais aussi productrice, ne se limite pas à un reflet, il maintient ses propres exigences qui ne sont pas celles du monde, mais celles de ses matières d'expression et du langage cinématographique. Il en est ainsi du corps spectatoriel pris ou épris dans l'étau de l'image. L'analyse doit en délier les composantes originelles narcissiques et lui libérer l'accès à d'autres espaces.

\section{— UN DOUBLE DE SIGNIFICATION}

L'imaginaire et son signifiant cinématographique dédoublent son sujet spectateur et son dispositif en une manière d'inclusion réciproque, où l' "expression synecdoque" de l'œil du sujet toutpercevant se fond aux mécanismes de projection écranique. Jean Louis Schefer dépeint l'anatomie d'un rapport spectatoriel dépositaire d'un double de signification. Le cinéma représente pour lui une expérience opaque et non transparente, primaire et 
non secondaire, appelant un retour certain aux origines des identités du Moi, déplaçant le centre des effets du film vers un intérieur virtuel des affects du spectateur. Ce dernier expérimente un second corps dans l'ignorance duquel il vit. L'Homme ordinaire du cinéma se projette totalement dans l'axe sémiotique d'un étrange désir d'extension du corps humain où l'effet de l'image est en deçà de sa figuration, de ses modèles, de ses représentations.

En un mouvement opposé, Christian Metz tente de dégager l'objet cinéma de l'imaginaire pour le conquérir au symbolique. "C'est dans le sillage [des opérations symboliques] que se situe l'espérance d'un peu plus de savoir, c'est l'un de ses avatars qui introduit au "comprendre ", alors que l'imaginaire est le lieu d'une opacité indépassable et comme définitoire" (1977, p. 10). Il y a chez Christian Metz un travail topique et dynamique du double, de dédoublement du sujet spectateur dans le dispositif cinématographique dans le double régime de vision et de croyance de sa visée filmique et de sa visée onirique.

Le corps de l'image filmique, comme réversibilité opérationnelle de l'image d'un corps d'un sujet versus une production artistique, engage un processus doublement imaginaire. Les prolongements cinématographiques des projections mentales de la surface du corps les dédoublent sur écran en représentations. Toutefois la parenté des concepts opératoires ne pousse pas l'analogie du corps propre du sujet et du corps du sujet spectatoriel jusqu'à l'identique, cette parenté nous pointe les dispositions du sujet à être en prise avec des forces antinomiques identitaires et elle nous livre un regard privilégié sur la posture spectatorielle apte aux renversements, aux dédoublements, à la réversibilité des rôles.

\section{Narcissisme}

Forces antinomiques et réversibilité des tensions, voie régrédiente du visuel, double de signification des deux corps spectatoriels selon Barthes et d'un corps autre chez Schefer, tous ces mécanismes tant de la perception du corps propre que du sujet spectatoriel disent les différentes couches entremêlées des identifications. Il n'y a pas une identification, il y a de l'identification avant 
toute chose. Étrangement ces processus se recoupent, se doublent et se dédoublent sous différents régimes perceptuels du monde, de soi et du film, jamais tout à fait les mêmes, jamais tout à fait différents, mais gardant toujours la mémoire de ce qu'ils ont été.

Le Moi est lié à l'image du corps propre. "L'image, c'est moi ", s'écrierait Narcisse tel l'enfant au miroir lacanien. Le narcissisme est une question d'identification par l'image avant d'être l'amour ou la haine de cette même image. "Le Moi n'est autre que cette captation imaginaire qui caractérise le narcissisme" (Nasio, p. 86). Comment ne pas se surprendre alors de la fascination de tout sujet pour les processus imageants?

"Le narcissisme secondaire se définit comme l'investissement libidinal de l'image du Moi, cette image étant constituée par les identifications du Moi aux images des objets" (Nasio, p. 83), et je souligne, non pas de l'identification aux objets tel le fait la libido d'objet opposée à la libido du Moi, mais bien à ses images. "Libido d'objet et libido du Moi ne sont toutefois pas en rapport d'exclusion: il existe une réversibilité de la libido" (Nasio, p. 82), tout comme il existe, comme nous l'avons vu plus haut, une réversibilité des images et des identifications, de leur retournement sur le Moi. Le narcissisme secondaire est une structure permanente du sujet, l'Idéal du Moi représente une formation narcissique qui n'est jamais abandonnée et aussi qui n'est jamais comblée.

Le Moi résulte de la sédimentation des investissements d'objets abandonnés. L'identification aux images de l'objet telle l'identification narcissique est une identification à un aspect partiel de l'objet visant à restituer au sujet sa part manquante au nom d'un idéal.

\section{- MÉLANCOLIE}

Le narcissisme secondaire du Moi est affaire d'image du Moi et du Surmoi. L'Idéal du Moi soutient le narcissisme. Le Moi ne peut être aimé et s'aimer que dans la mesure où il satisfait certaines exigences. Cette affaire d'image est duelle entre le plaisir de se voir et le devoir d'animer cette image à la loi, à l'éthique. Il ne faut pas se surprendre alors des aspects négatifs liés au narcis- 
sisme. Aux aguets, le Surmoi met le Moi à l'épreuve de ses idéaux et projette dans sa perception du réel ses incomplétudes, ses insatisfactions, ses plaintes. L'identification narcissique se retourne alors en identification accusatrice. Car là où s'abat le Surmoi, s'érige tout un dispositif d'observation, de conscience critique. Cette image du Moi, de moi, sera-t-elle la bonne, propice à la projection collective et à mes petites différences? Si tout narcissisme secondaire ne conduit pas nécessairement à la mélancolie, tout travail de mélancolie recoupe toutefois la voie régrédiente du narcissisme. Le texte "Deuil et mélancolie" (Freud, 1983) est inséparable de "Pour introduire le narcissisme" (Freud, 1982). La mélancolie emprunte une partie de ses caractères au deuil et l'autre partie au processus de la régression à partir du choix d'objet narcissique. L'identification narcissique secondaire se fait rarement sous le couvert de la bonne relation d'objet, son travail s'avère nettement plus laborieux sous la captation imaginaire de l'Idéal du Moi et du Surmoi tant le réel ne répond plus de cette image. En ce sens, nous sommes loin de l'image de toute-puissance originant du narcissime primaire. Dans le cas de la mélancolie, l'identification narcissique du Moi à l'objet perdu consistera donc à retourner cette perte sur le Moi afin de maintenir intact imaginairement la présence de l'objet perdu. Du narcissisme et de la mélancolie, l'on dénotera au niveau du processus ces mêmes qualités de retournement et de réversibilité pour s'approprier l'objet et surtout cette même capacité de captation imaginaire.

\section{- CINÉMA}

Jean-Louis Baudry insiste sur le caractère artificiel du cinésujet, pure production du dispositif cinématographique qui favorise cette construction et cette position. Il émet l'hypothèse d'un dispositif qui déterminerait artificiellement cet état régressif du sujet spectateur, d'un retour vers un narcissisme relatif (p. 44) afin de satisfaire dans le désir de cinéma et le plaisir qu'il y trouve cet état antérieur, mais maintenant perdu. Et pour Metz, c'est dans cette emprise même du signifiant imaginaire du cinéma que le spectateur s'identifie primairement à lui-même comme pur acte de perception (1977, p. 69). Le spectateur aime 
se percevoir, s'aime percevoir, il est pris en flagrant délit d'autoamance de perception.

Premièrement, le spectateur entretient avec les films de véritables relations d'objet, relations fantasmatiques bien distinctes des relations réelles et des objets réels. Deuxièmement, il s'agit bien ici d'une relation imaginaire et troisièmement, cette relation imaginaire opère sur le mode de l'absence (Metz, 1977, p. 62-65). Ces trois données viennent qualifier la perception au cinéma d'un enjeu imaginaire assez troublant entre présence et absence allant au-delà d'une simple simulation d'impression de réalité. En cela, le "corps spectatoriel du cinéma" se trouve être pris entre "narcissisme" (comme sujet tout-percevant dans la complexité désirante de sa perception entre projection et introjection de l'image des objets) et entre "mélancolie» dans la captation imaginaire et incorporante de la voie régrédiente de "l'identification narcissique " à l'objet perdu.

En ce sens, le film est une impasse et il est loin d'être le lieu de toutes les résolutions.

\section{À tout prendre de Claude Jutra}

La question de l'identité dans $A$ tout prendre (1963) rejoint toutes les tensions entre réel et imaginaire sous le couvert de l'image et l'enjeu de sa représentation filmique en un corps fragmenté, discontinu. J'ai choisi ce film parce que Claude, le personnage principal, est le spectateur de ses propres représentations: narcissisme du film, narcissisme dans le film, narcissisme du cinéma.

Les syntagmes en accolade du film traduisent bien l'éclatement de ces postures comme figures, comme images dans leur élaboration et leur possible liquidation. Ce segment a-chronologique, Christian Metz l'a défini dans sa grande syntagmatique comme " $[\ldots]$ une série de brèves scénettes représentant des événements que le film donne comme des échantillons typiques d'un même ordre de réalités [... dans] leur parenté supposée au sein d'une catégorie de faits" (1971, p. 127). Cette multiplicité de plans se perçoit et se conçoit ici dans son ensemble discursif, cette structure de montage traduit parfaitement le questionnement identitaire de Claude: quelle image de moi est la bonne? 


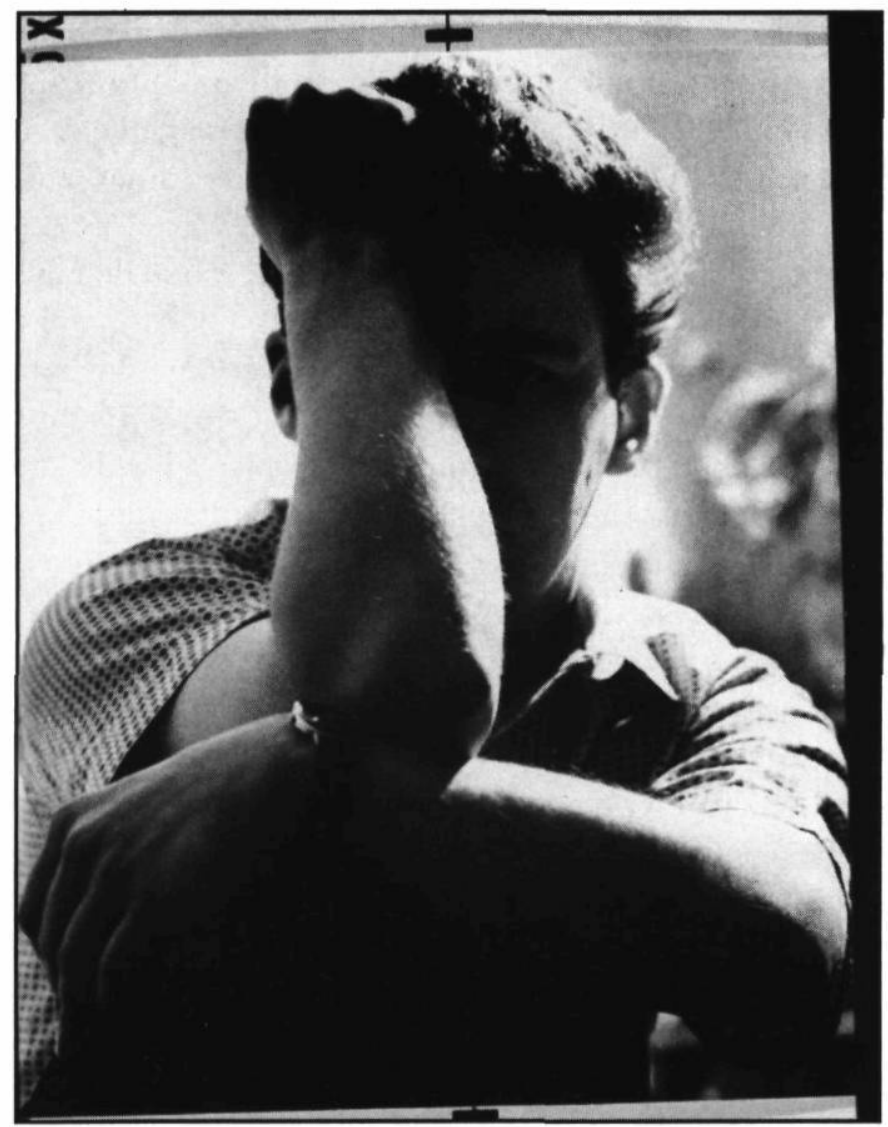

\section{À tout prendre de Claude Jutra (1963)}

Collection Cinémathèque québécoise

Au tout début du film, Claude semble reculer à l'idée de se rendre à une fête organisée par des amis, il n'en apporte pas moins de méticuleux soins à sa propre préparation. Il enfile sa chemise devant un miroir, nous le voyons en plan demiensemble et l'entendons se dire en voix off: "Je me dérobe toujours. Ah! je suis jeune, j'ai le temps. Ma jeunesse», il éclate alors d'un fou rire désinvolte et la caméra en un "zoom" avant saisit dans le miroir un gros plan de Claude pris dans ses pensées: "Me débarrasser à jamais de tous ces personnages en moi qui sont ce que je ne fus jamais et qui me hantent." Il s'ensuit une série d'images de Claude posant devant le miroir premièrement 
habillé en rocker conspuant le monde entier pour se venger d'avoir été mis au monde, puis vêtu élégamment comme un hautain aristocrate, ensuite costumé en un Pierrot magique et lunaire et enfin accoutré d'une veste de cuir et de verres fumés, arrogant, il tire du fusil sur le miroir qui se fragmente, éclate, ne réfléchit plus, se tait. Le segment se boucle avec le plan initial de Claude se préparant pour sa sortie vêtu d'un complet-veston, digne image d'un artiste de sa décennie, image, nous le verrons plus tard, qui ne saura elle aussi résister à son effondrement. Ainsi à la toute fin du film, il y aura reprise et continuité de ce plan final du segment en accolade. Or à la lecture de la lettre d'abandon de Johanne, il récite en voix off les mots de sa bien-aimée: "Sois en paix, tu n'as laissé aucune trace", mots auxquels une deuxième hauteur de la voix off de Claude encore se superpose comme en écho d'un cri de la conscience: "Elles sont en toi, les traces." La caméra glisse sur la lettre et le plan suivant rappelle ce dernier plan du syntagme en accolade ci-haut décrit de Claude en complet-veston; un "zoom" avant se précipite sur l'image du miroir où il noue sa cravate, un coup de feu éclate, la glace s'étoile, cette dernière image de notre personnage s'étiole elle aussi, ne tient plus. À première hauteur du commentaire interne, Claude constate, étonné, en voix off: "Ça alors, c'est fait", à deuxième hauteur de la voix off, il assène un vengeur: "C'est bien fait."

L'identification dans le travail de mélancolie, selon Benno Rosenberg, peut se vivre comme moyen de transition pour revenir à l'objet, une manière de bénéfique introjection pour se sortir d'une situation intenable en passant par l'investissement narcissique. De ce point de vue, la notion passive de repli ne tient plus bon, Rosenberg construit autour de la notion d'identification dans la mélancolie la dynamique de travail psychique. Il en souligne deux grands traits constitutifs obligés: le fait élaboratif de compulsion de répétition et l'idée même de la liquidation de l'identification comme investissement narcissique d'objet.

Mais ce que le travail de mélancolie nous montre, c'est une situation où l'identification reste la seule possibilité d'élaboration. De ce point de vue, le travail de mélancolie est une expérience cruciale où l'identification est la seule à l'œuvre, seule capable de fournir une voie de 
sortie au moi coïncé entre l'impossibilité de désinvestir l'objet et l'impossibilité de continuer à l'investir (Rosenberg, p. 1529).

L'élaboration et la liquidation de l'identification narcissique en cours dans le travail de mélancolie recouvrent donc le travail du film dans le syntagme en accolade analysé et décrit plus haut. L'élaboration est affaire de transition, la liquidation tient lieu de métamorphose. La chrysalide couve le prisme de toutes les reconstitutions à venir. Les commentaires de Claude Jutra dans son Manifeste (1963) pour le film $\grave{A}$ tout prendre recoupent cette même conception de circulation dynamique du réel à l'imaginaire, de la projection à l'introjection, pour s'assurer une voie de sortie symbolique.

Mon miroir sert de cadre aux déguisements nombreux que j'aime revêtir. Au cours d'une métamorphose, je brise le miroir avec une arme d'assassin. Il se reconstitue aussitôt, mais à l'instant précis où je le quitte, où je lui dérobe mon image véritable pour m'acheminer vers mon destin, c'est-à-dire la fête où je rencontrai Johanne, où je succombai à l'amour.

Un autre segment de même configuration en accolade est aux prises non plus avec la représentation d'images anciennes qui viennent hanter l'esprit de Claude et dont il a voulu se débarrasser, mais avec la vision et la prévision d'images à venir dans lesquelles il s'est imaginairement et comiquement empêtré. Claude en fait une histoire, une question de représentation.

Le segment en accolade intervient, suite à ce point de vue sur la représentation des choses, juste après une discussion à la terrasse d'un café où Claude est en compagnie de Victor, son ami de théâtre, qui lui avait jadis reproché sa vision $16 \mathrm{~mm}$ des choses. Fort à propos, assis à la table du café, Claude songe, ébranlé, à la précédente confession de Johanne: du pur cinémavérité où elle lui a avoué difficilement et dramatiquement son identité d'orpheline québécoise et non de belle noire de l'exotique île d'Haïti. Victor laisse aller les choses, le silence d'une présence, et puis interroge Claude, par la lecture à voix haute d'une citation de Proust interposée, sur la réalité des choses: "Ce que 
nous appelons la réalité est un certain rapport entre ces sensations et ces souvenirs qui nous entourent simultanément, rapport que sait créer une simple vision cinématographique laquelle s'éloigne par là d'autant plus du vrai qu'elle prétend se borner à lui." L'homme de théâtre qu'est Victor cherche à provoquer Claude, l'homme de cinéma.

L'homme de cinéma lui rétorque par le plaisir du film en une moquerie élégante et théâtralisée de la représentation et lui démontre et montre non seulement comment il se voit, mais aussi comment il se joue. Il saisit l'occasion de ce segment en accolade pour affirmer que la représentation cinématographique ne le lie aucunement à une vision réaliste en $16 \mathrm{~mm}$ des choses et que tout est affaire d'images. La voix off de Claude ouvre le segment, elle implante une lecture documentarisante par la tonalité de la voix et puis fictivisante par la teneur de son contenu et la théâtralité progressive de son jeu. Le cadrage fixe et frontal en plans d'ensemble sur fond dénudé d'un très haut mur de pierres et ce, pour toutes les scénettes du syntagme, contribue à donner de cet espace filmique l'impression d'une scène de théâtre sur laquelle Jutra va interpréter ses différentes métamorphoses en effectuant systématiquement et linéairement ses entrées et sorties de champ de gauche à droite ou de droite à gauche des plans. Le champ est vide et sa voix de commentateur externe maintenue tout au long du segment inaugure la première scénette: "Pour la bonne intelligence de ce récit, je dois l'interrompre un instant afin d'exposer quelques faits", alors Claude traverse le champ de gauche à droite, nonchalant, suffisant, tergiversant, et ce commentaire l'accompagne: "Voici comment marche un jeune bourgeois, déjà plus très jeune, déjà un peu trop gras, et qui cherche à placer quelque part ses restes d'enthousiasme", et puis il parcourt le plan en sens inverse de droite à gauche, plié, ralenti, recroquevillé, sous ces mots de désillusion : "Comment il se voit d'ici quelques années. "Se succèdent alors une série de propositions dans le même cadrage de plan, la même position énonciative du commentaire de la voix off, la même mise en scène du jeu et des déplacements dans la traversée du champ, le cumul des scénettes et l'effet de répétition en assurent la drôlerie. Sous la voix: "Habité par de gran- 
des idées", l'image: il longe littéralement le mur. "Porté par les événements", il tient les guidons d'une bicyclette. "Paranoïaque", il marche avec des contorsions expressionnistes. "Poète", il flotte la tête dans les nuages. "Quand il se trouve en difficulté...un regard à l'Est... un regard à l'Ouest... Statu quo", il titube. "Et parfois pour amuser les enfants", Claude a quitté l'enceinte du mur pour se retrouver dans une aire ouverte, une rue avec des enfants enjoués; le segment se ferme avec ces images sur ce dernier commentaire: "Fin du documentaire. Place au vrai cinéma."

Cette question de représentation soulevée par ce syntagme en accolade dédoublera dans le plan suivant le même renversement de régime dans la substitution des termes croisés. Ce vrai cinéma fait place aux pures techniques du cinéma direct pour décrire l'ambiance d'un studio de tournage, le réalisme et la technicité de son appareillage. Cette idée d'écriture dans le renversement des formes du documentaire à la fiction, de la fiction au documentaire, tout en en maintenant les dualités, ces possibilités d'échange dans le retournement narcissique des identifications, tout en en assurant les contradictions dynamiques de leur élaboration, se dédoublent dans la mise en abyme du film, après la confession de québécité de Johanne et après l'aveu d'homosexualité de Claude, lors du tournage dans le studio de ce petit dilemme mélodramatique de la rupture d'un couple mis en scène par Claude, le personnage-réalisateur. Face à face, Monique et François concluent leur mésentente; elle lui dit: "Ce n'est pas moi que tu aimais, c'est une image", aussitôt lui retourne-t-il la proposition: "Non, c'est toi parce que tu me délivrais de toutes les images."

Ces deux petites phrases contiennent en elles-mêmes les traits de l'élaboration et de la liquidation des identifications narcissiques du travail de mélancolie mis en jeu dans la représentation filmique. Les échanges dans la transition des identifications narcissiques passent obligatoirement par Johanne, dans la captation imaginaire de l'Autre, dans les nombreux attraits et retraits spéculaires d'un Moi projeté et défensif, puis introjecté et retourné sur soi. Une séquence par épisodes prélude de l'échange narcissique des amoureux dans le rituel des cadeaux chez les nouveaux 
amants où, dans le retournement libidinal économiquement investi, c'est celui qui donne qui reçoit. Petite boîte à musique, coupe, coffret de dés, bracelet, miroir, appareil-photo jouet, ces objets en plans rapprochés passent de jour en jour, de main en main, miroitant d'amour et d'auto-amance dans cette cérémonie sacrée et scandée par les louanges de Claude toujours en voix off: "Acheter, posséder, quel plaisir! Partager dédouble ce plaisir. Offrir un objet, c'est l'acquérir deux fois. L'offrir à la personne aimée, c'est le céder sans y renoncer. Cadeau qui souvent satisfait davantage le donneur que celui qui reçoit. Johanne et moi pratiquons furieusement le rituel du cadeau."

De Johanne à Claude, il y a de part et d'autre usurpation dans l'échange d'images. Sans cesse mise en représentation dans les parades de mode et mannequin photographié, se mettant souvent en scène par la danse et la chanson, Johanne n'est Autre que comme modèle idéalisé d'une surface narcissique retournant la propre blessure de Claude. Étrangère dans son propre pays et (parce que) orpheline: "Je suis pas Haïtienne du tout, et je suis obligée de dire ça... Ça fait depuis longtemps... Et je suis née ici, je suis une orpheline..." ", différente et à la fois si semblable, la confession hésitante et titubante de Johanne, en plans rapprochés et bougés typiques du cinéma direct, concerne son besoin d'identification: "M'identifier...quelque chose de..." " et en tant que tel, elle ne peut que révéler de Claude son étrangeté à lui-même. La confession de Johanne déclenche l'aveu de Claude, l'aveu de Claude s'imbrique dans la confession de Johanne, le retour infini des images fait éclater le miroir des identifications. La position amoureuse de Claude, de glorieuse qu'elle était, s'avère désormais souffrante, douloureuse.

Toute cette agressivité versée à son compte le place dans une situation désirante masochiste. L'amour lui fait mal, et avant toute chose, ce sont les caresses de Johanne qui l'abîment et qui se transforment à ses sens en douleur. En plan demi-rapproché, Johanne, la tête de Claude sur son épaule, lui effleure les cheveux, le front, doucement et lentement, et Claude, piégé dans cette "prison d'or et de cristal" des tendresses de Johanne, se plaint en lui-même: "Gestes habiles mais aussi cruels. Comme si elle fouillait une plaie avec ses doigts. Étrange mélange de 
délice et de supplice. "Et dans une offensive tout empreinte de sérénité et de crainte mêlée, Johanne lui murmure la question finale: "Mamour, aimes-tu les garçons? " Un "zoom" avant plonge en très gros plan sur ce front agressé par des doigts amoureux et Claude, à la suite d'un bruit off de miroir brisé, se répond, se livre et se délivre intérieurement: "Je ne dis pas oui pas plus que je ne dis non. Ainsi s'échappe le secret que je séquestrais depuis des temps plus lointains que mes premiers souvenirs ", et la voix enchaîne au plan suivant sur le plateau de tournage, sous la lumière des projecteurs: "Johanne a fait cela. De ses mains de femme, elle a ramassé le plus lourd de mon fardeau. Elle m'a fait avouer l'inavouable et je n'ai pas eu honte et je n'ai pas eu mal. Et maintenant tout est changé, car cette impérieuse aspiration jamais assouvie, de tourment qu'elle était, a pris la forme d'un espoir."

Lélaboration et la liquidation des identifications dans la dialectique interne du travail de mélancolie passent par des pulsions de destructivité pour conduire à une certaine libération, délivrer Claude de toutes ses images. Le choix narcissique d'objet dans l'homosexualité peut aussi se poser en termes de bonne introjection, car entre se fermer sur soi et s'ouvrir à soi-même dans le processus d'introjection-identification, " [...] c'est la balance économique entre ce qui est gagné et ce qui est perdu qui décide en dernière instance de la réussite ou de l'échec du travail de mélancolie" (Rosenberg, p. 1535). L'homosexualité avouée dans $A$ tout prendre a beaucoup contribué à l'enfermement du film sous deux couverts: un enfermement théorique tributaire d'un concept univoque et figé du narcissisme et un enfermement autobiographique par l'éclat même de ladite révélation. Bien sûr, le contexte autobiographique semble tout légitimer par la présence de Jutra comme auteur-narrateur-personnage du film, mais il n'en justifie pas le cloisonnement autour de la personne du réalisateur. Tout en le privant, disait-il, de son droit au jeu, cette même attitude nie au film son travail signifiant. Car pour revenir à notre point de départ, le risque passe par les inclusions diverses et réciproques du désir, de l'art, du cinéma, et comme l'a écrit Dominique Noguez au sujet du film $A$ tout prendre: "Ce jeu a toutefois quelque chose de moins 
gratuit et de plus dangereux qu'une simple reconstitution: il a l'incalculable importance d'un récit psychanalytique" (p. 106).

Dans un article éclairé sur la rhétorique de l'homosexualité dans $\grave{A}$ tout prendre, Alain-Napoléon Moffat voit dans le contexte autobiographique du film un grand potentiel d'altérité et de jeu ouvert dans son auto-interprétation. À ses yeux, «[...] ces cassures d'unité dans le personnage de Claude et qui sont autant de coupures diégétiques dans le film" (p. 8) engendrent ce dilemme de la représentation. De ce point de vue, l'autoportrait remplit mieux la commande du film par la discontinuité et la brisure de ses traits et ce, en opposition au réglage narratif d'un récit linéaire et chronologique. Cette rhétorique tend à déstabiliser "la notion spéculaire traditionnelle de l'homosexualité" et cherche à ouvrir la problématique "vers le lieu d'une personne (ouverte, désirant espace de l'autre), comme un enjeu de création" (p. 11). Quant à nous, l'identification narcissique dans l'homosexualité nous intéresse, car elle met en relief dans le travail dynamique de mélancolie une autre piste que l'agir dépressif. Elle ébauche sur son axe, comme passage d'une régression de l'investissement d'objet, une possibilité d'élaboration dans la dialectique de ses constructions et de ses destructions vers une libération de l'accès mélancolique. Le texte de Rosenberg nous en aura préparé une voie de sortie.

La communauté connue de type d'investissement narcissique d'objet entre les homosexuels et les mélancoliques fait que l'homosexualité peut avoir une importance capitale: il nous semble que cela fait partie du traitement du mélancolique que l'homosexualité (manifeste ou non) puisse représenter l'axe par lequel nous pouvons faire évoluer l'investissement narcissique d'objet vers un investissement objectal proprement dit. L'homosexualité étant en analyse l'une des voies royales de la régression de l'investissement d'objet au narcissisme, elle peut aussi être la voie de "retour ", la voie d'objectalisation du type d'investissement (p. 1528).

Claude, à la toute fin du film, avant de décider de partir, de changer d'endroit, de paysage et de visage, s'avance sur une jetée qu'il arpente jusqu'à son extrémité. Il plonge dans la rivière pour 
aussitôt en émerger, ressuscité, flottant dans les cieux. Sous le mode de l'humour et de la désillusion, le plan est repris deux fois, comme une sorte de reprise de l'imaginaire pour en désavouer les eaux profondes et les voies éthérées. Claude y récite un texte poétique, d'abord lentement et puis rapidement, sous le thème de la mélancolie.

Un jour, je m'en irai mélancolique et sombre, au bout de la jetée entre le ciel et l'eau, lorsqu'enfin la lumière a triomphé de l'ombre et que l'espace est plein de cris de mille oiseaux. Ainsi d'un pas prudent, marchant entre deux longes, j'avancerai sans bruit, suspendu au milieu, hésitant un instant entre l'azur et l'onde, puis je prendrai mon vol en direction des cieux.

Le film (tout film), ai-je écrit plus haut, est une impasse, il est loin d'être le lieu de toutes les résolutions, et j'ajouterais maintenant qu'il est bien près d'être le lieu de toutes les identifications, positives et négatives, interchangeables et réversibles à souhait grâce à la position psychique narcissique du spectateur.

Les gender studies et les cultural studies ont poussé à leur dernier retranchement l'identification des différences, de différences sexuelles, sociales, réelles certes, mais de différences émolliées, arrondies, traversées régressivement par des pulsions contraires. Lidentification du spectateur est pluristratale, et ses strates m’apparaissent beaucoup plus entremêlées que hiérarchisées et ce, malgré l'idéologie et les stéréotypes dominants.

Le cinéma met à l'épreuve les points de vue de la différenciation (en ce qui a trait à l'identification spectatorielle) trop uniformes et logiques, voire systématiques dans leur raisonnement binaire. Il fait la preuve qu'il n'y a pas un pôle identificatoire, fixe, systémique, mais plutôt une instance identificatrice définie par sa polarité ambivalentielle, ambivalences certes liées aux spécificités multiples des différences sexuelles, sociales, nationales, mais ambivalences aussi narcissiquement investies dans le dispositif même du cinéma. Lesquelles de ces ambivalences se présentent perceptivement les premières? Aucune, puisqu'elles composent le treillis de la tessiture identificatrice, retournant sans cesse leurs figures.

Université du Québec à Chicoutimi 


\section{B I B LIOGRAPH IE}

Barthes, Roland. “En sortant du cinéma ". Communications, n” 23 (1975) p. 104-108.

Baudry, Jean-Louis. L'Effet Cinéma. Paris: Albatros, 1978.

Brady, James. "À tout prendre: fragments d'un corps spéculaire". Copie Zéro, n" 37 (octobre 1988) p. 23-26.

Freud, Sigmund. La Vie sexuelle. Paris: P. U. F., 1982.

Freud, Sigmund. Métapsychologie. Paris: Gallimard, 1983.

Green, André. Narcissisme de vie, narcissisme de mort. Paris: Minuit, 1983.

Jutra, Claude. Manifeste. Montréal : Cinémathèque québécoise, 1963-1964.

Leclaire, Serge. Démasquer le réel. Paris: Seuil, 1971.

Metz, Christian. Essais sur la signification au cinéma. Tome I. Paris: Klincksieck, 1971.

Metz, Christian. Le Signifiant imaginaire. Paris: Union générale d'éditions, 1977.

Moffat, Alain-Napoléon. "À tout prendre" de Claude Jutra, une théorique de l'homosexualité. Montréal: Cinémathèque québécoise/Association québécoise des études cinématographiques, 1991.

Nasio, J.-D. Enseignement de sept concepts cruciaux de la psychanalyse. Paris: Rivages, 1989.

Noguez, Dominique. Essais sur le cinéma québécois. Montréal : Éditions du Jour, 1970.

Rosenberg, Benno. "Le travail de mélancolie ou la fonction élaborative de l'identification ou le rôle du masochisme dans la résolution de l'accès mélancolique». Revue française de psychanalyse, tome I, n" 6 (novembre-décembre 1986).

Sani-Ali. Corps réel. Corps imaginaire. Paris: Dunod, 1977.

Schefer, Jean Louis. L'Homme ordinaire du cinéma. Paris: Gallimard, 1980.

Schilder, Paul. L'Image du corps. Paris: Gallimard, 1968. 